\title{
Automated Verification of Structural Engineering Assembly using Convolution Neural Network
}

\author{
S.Padmashree, Sushma.S.J
}

\begin{abstract}
Artificial Intelligence has mostly penetrated in every field of technology and our lifestyle in numerous ways. The contribution of $A I$ in the field of Civil engineering which mainly focuses on planning, design and construction is enormous. The main objective of this work is to develop a system that will automate the process of detecting errors in the engineering plans or drawings of structures. The work adapts convolution neural network technique with the help of Inception V3 model to automate detecting of multiple errors using Artificial Intelligence. AI technique has proven to be more effective, accurate and less time consuming against the existing manual verification technique.
\end{abstract}

Keywords : Artificial Intelligence, Structural assembly, Deep Learning, Convolution Neural Network

\section{INTRODUCTION}

Artificial Intelligence (AI) is a way of making a computer-controlled robot, or a software think intelligently, in the similar manner as to how the human intelligence works. Artificial intelligence is a branch of computer science, involved in the research, design, and application of intelligent computer. It has become increasingly important in the modern world from search engines, video games, and financial algorithms to autonomous vehicles to provide useful services. This project also inculcates Image Processing Techniques which is one of the core domains of Electronics and Communication. Image processing is a method to perform some operations on an image, in order to enhance image or to extract some useful information from it. The civil engineering sector is highly dependent on structural assembly drawings. A structural assembly drawing is a type of engineering drawing it is a plan or set of plans for how a building and structure should be built. Structural assembly drawings are generally prepared by registered professional structural engineers. The traditional and current methodology adapted for transforming a structural design to an actual structure or a building consumes a significant amount of time. Errors or irregularities

Revised Manuscript Received on June 30, 2020.

* Correspondence Author

Dr. S. Padmashree*, Professor, Department of Electronics and Communication Engineering, GSSS Institute of Engineering and Technology for Women, Mysuru, India. Email: padmashree@gsss.edu.in

Sushma S J, Associate Professor, Department of Electronics and Communication Engineering, GSSS Institute of Engineering and Technology for Women, Mysuru, India Email: sushmasj@ gsss.edu.in

(c) The Authors. Published by Blue Eyes Intelligence Engineering and Sciences Publication (BEIESP). This is an open access article under the CC BY-NC-ND license (http://creativecommons.org/licenses/by-nc-nd/4.0/) present in the structural design itself could lead to a lot of complications since it is done manually. Thus this project aims to automate the verification of structural assembly drawings using artificial intelligence and image processing techniques. The objective of the paper is achieved in two stages, the first one is using image processing techniques to extract desired data from the structural drawings using Python platform and Optical Character Recognition. The second stage uses a very effective domain of AI that is Convolution Neural Network (CNN) in order to verify the Structural Assemblies. AI helps in automating data collection and data analysis techniques along with Image Processing to improve several aspects of construction engineering and management for productivity assessment, safety management, idle time reduction, prediction, risk analysis, decision-making and optimizing construction costs.

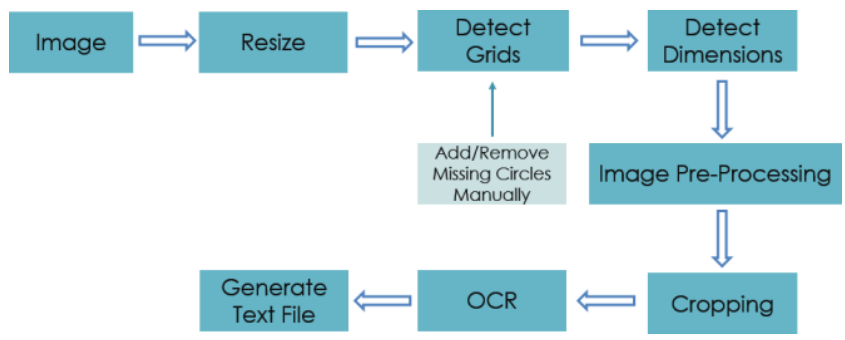

Figure 1.1 Overview of the proposed work

In this paper two segment approaches are undertaken, first segment is structural assembly drawing verification using image processing and the second segment is structural assembly verification using convolution neural networks. The drawings undergo few image processing techniques to achieve feature extraction. The output obtained from the image processing techniques is fed into the optical character recognition. The result of the OCR are obtained in a text file. The text file gives the dimensions between two consecutive grids. This is shown if figure 1.2 In the second segment a convolution neural network classifier is built on inception V3 model based on the technique of transfer learning shown in figure 1.3 The classifier model is trained and tested with various structural assembly images. In order to make it accessible to the user, the program is deployed onto a web portal. The web portal is designed with a feature to upload images, on uploading of structural assembly images the classifier classifies the assembly images into correct assembly or error in assembly. Along with the classification of assembly image the web portal also states a confidence score of around 0.69 with respect to the training images.

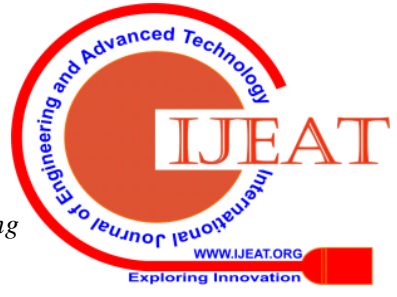




\section{METHODOLOGY}

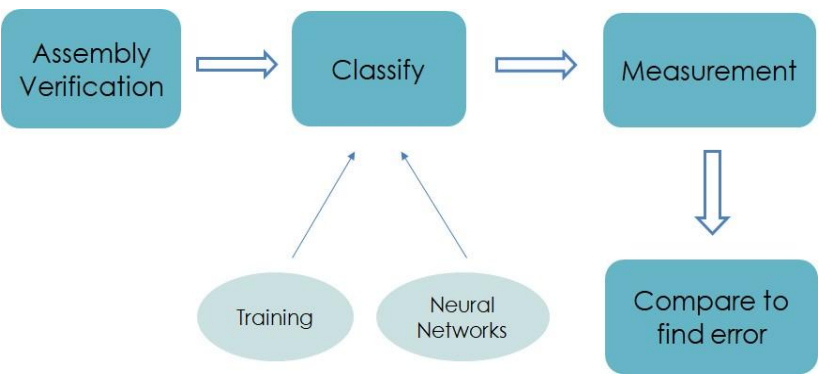

Figure 1.2 Block diagram for Image processing segment

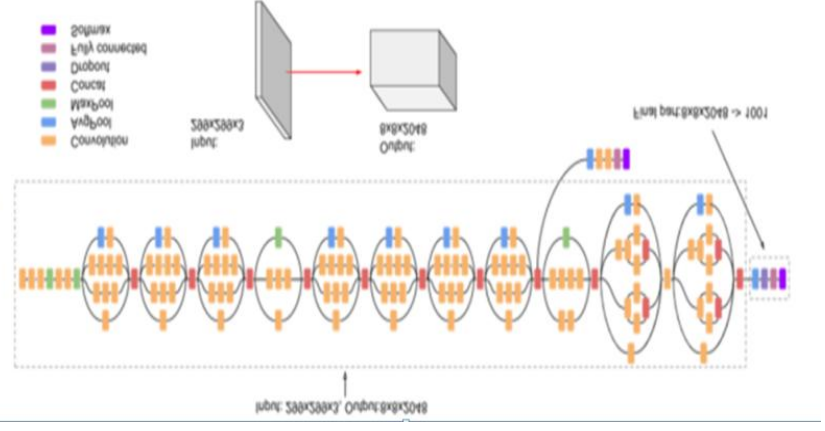

Figure 1.3-CNN V3 inception model

The objective of verifying structural assemblies is to check whether the assemblies are placed correctly and this is done by using inception v3 model shown in figure 1.3 and altering its last layer accordingly to train the layer with the images collected of structural assemblies.

\section{LITERATURE SURVEY}

The paper deals with artificial intelligence and its sub domain hence the reference of many papers related to the field were taken

Tianmei Guo et.al [1] in their paper has used a simple convolutional neural network to implement image classification at a low cost. On the basis of the working of convolutional neural network , Different optimization algorithms were studied to find an optimum method for image classification.

Belen Ferrer et.al[2] in their paper has discussed a Noninvasive image based method which is a normalized experiment with safety norms in building construction have been discussed.

Pavol Bezak [3] in his paper has proposed a model which is capable to recognize the right object in the photographs of various historical buildings in the town The model designed was scalable and was able to learn more images from more datasets. omplex dataset of photographs of various historical buildings taken from various angles was considered to be the base for perfect validation..

Arthur Shi Xiao-Hua Yu [4] proposed a novel NDE (Nondestructive Evaluation) method for SHM based on Discrete Wavelet Transform (DWT) and Artificial Immune Systems (AIS).. Artificial Immune Systems could be trained by the "normal" data of buildings and there is no need to destroy the building so as to identify measurements of "abnormal" data for training, however, its performance is often limited by the curse of dimensionality issue.
A classifier is trained and built on the inception v3 model with the help of transfer learning approach. The Inception V3 model is simple classifier and a pre-trained model that can classify images into 1000 classifications. The data base used for the training is taken from image net . The model is chosen for the current work as it is easy and available Inception V3 is the third version after Inception V1 and Inception V2. The error rate of Inception V3 model was 3.5\%. Transfer learning is one method used in deep learning. It basically makes use of auxiliary data to being trained with the existing model to help the new model achieve a better understanding of new image dataset and have improved results. Therefore, the auxiliary data and the target data supposed to be commonly related to each other. Figure 3.1 shows the idea of transfer learning. Transfer learning provides extra bonus over CNN with regard to time and accuracy.

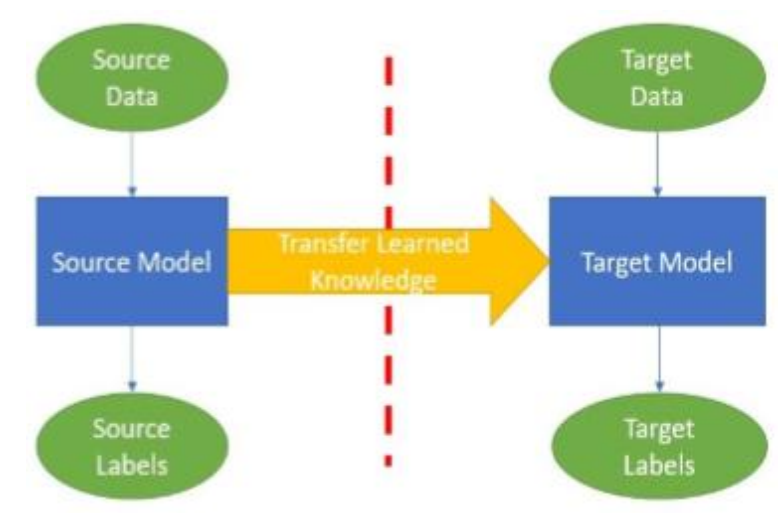

Fig 3.1: Transfer learning concept.

\section{Data set:}

The dataset used for this paper consists of around 200 assembly images. Prior to shipping the steel columns and beams to the construction site they are temporarily assembled for verification. The images of this assembly are captured and taken as dataset for building the classifier on inception v3 model as shown in figure 3.2

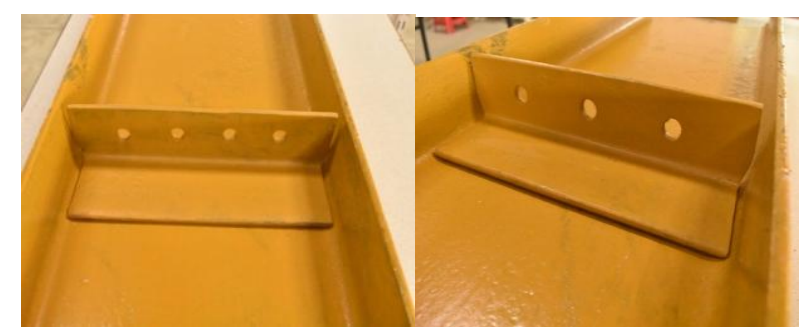

Figure 3.2: Assembly images.

Fig 3.2 depicts the assembly images taken in various angels. These images are then used for training and testing the CNN classifier.

Proposed approach:

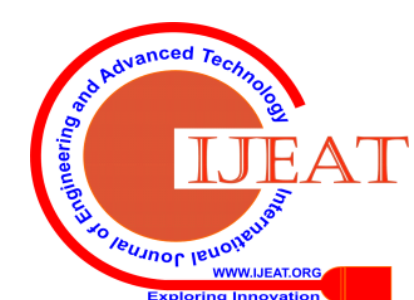




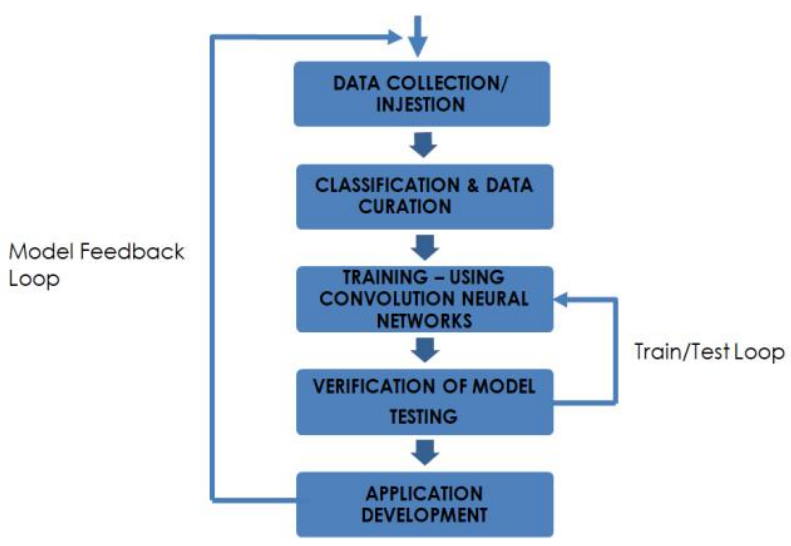

Fig 3.3: Methodology for CNN model.

\section{Data Collection:}

Data is collected from the data set which involves around 200 images of the assembly in real time. The images involve various assemblies and components taken in various angles. Figure 3.4 depicts the input data set.

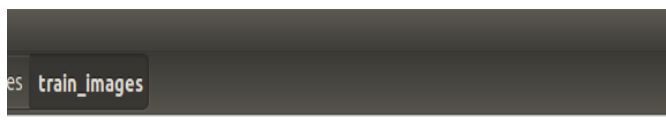

Input Images

Figure 3.4: Data set

\section{Data Curating and Classification:}

On completion of the data collection and storing.,the data is curated, in order to remove blurred images and certain images where the components can't be identified. Thus data curation is a must. After curating of the data the classifier need to be built using training images and training features which define the classification.

\section{Training}

The basis of any classification lies in the training part of the methodology. Training features are extracted from the images and are set as training labels. Based on the training labels the data set are classified. Fig 3.4 shows the training of CNN model in the command prompt.

\section{Testing}

Testing involves verification of the classifier. Thus a random assembly image is used for testing the classifier. This process in done in loop to attain accuracy stated as Train/Test loop. The output of the testing phase is shown in Fig 3.5.

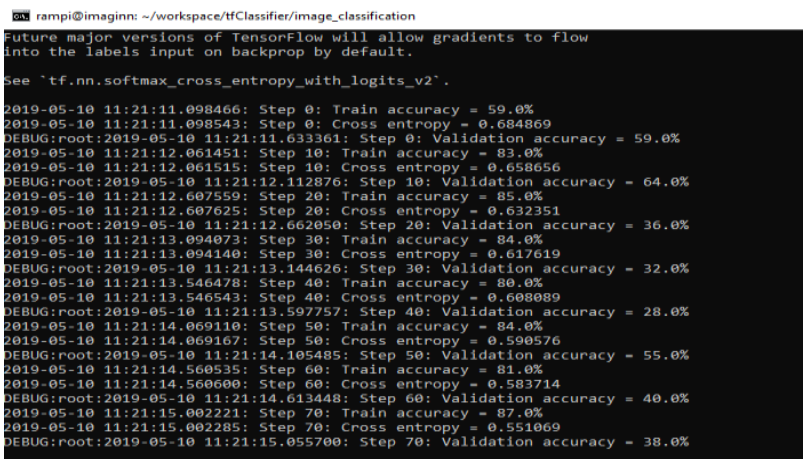

Figure 3.4: Training of CNN model with data set.

The CNN classifier model built is deployed onto a web portal to make it accessible. The web portal is deployed with a feature to upload the assembly image. After the assembly image is uploaded the CNN model classifies it and states on the Web portal whether the assembly is correct or there is error in assembly.

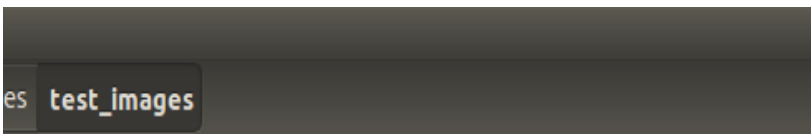

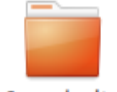

four bolts

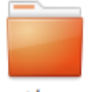

others

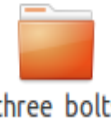

three bolts
Figure 3.5 output data set

\section{RESULT:}

The main results of this work are carried out in two segments. The results obtained in the first segment that is, Structural Assembly Drawing Verification is performed using Image Processing techniques and is explained in steps shown in figure 4.1. This segment is carried out using PYTHON programming for the verification of structural engineering assembly drawings. This part uses few image processing techniques to attain high accuracy results in minimal time and expenditure along with the assistance of Optical Character Recognition (OCR) to extract the desired output

- Resizing

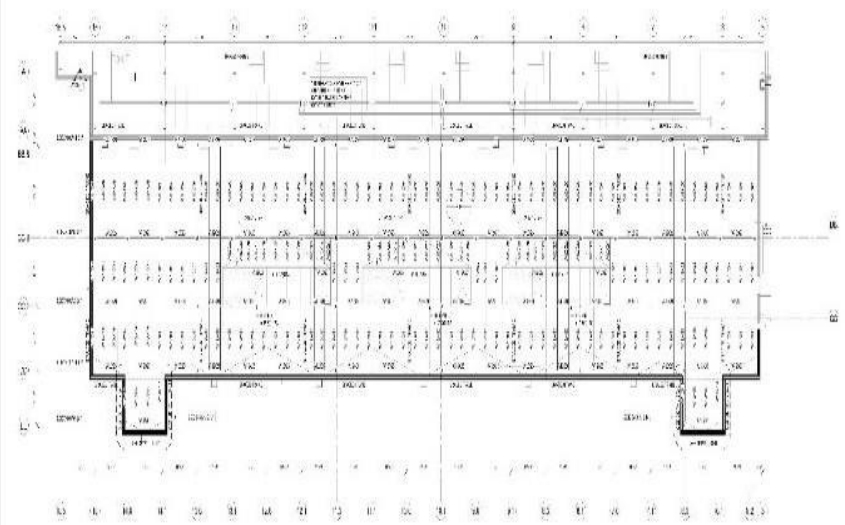

Published By:

Blue Eyes Intelligence Engineering

\& Sciences Publication

(c) Copvriaht: All riahts reserved.

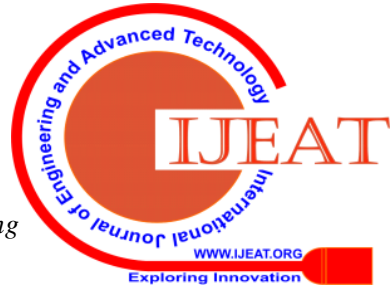



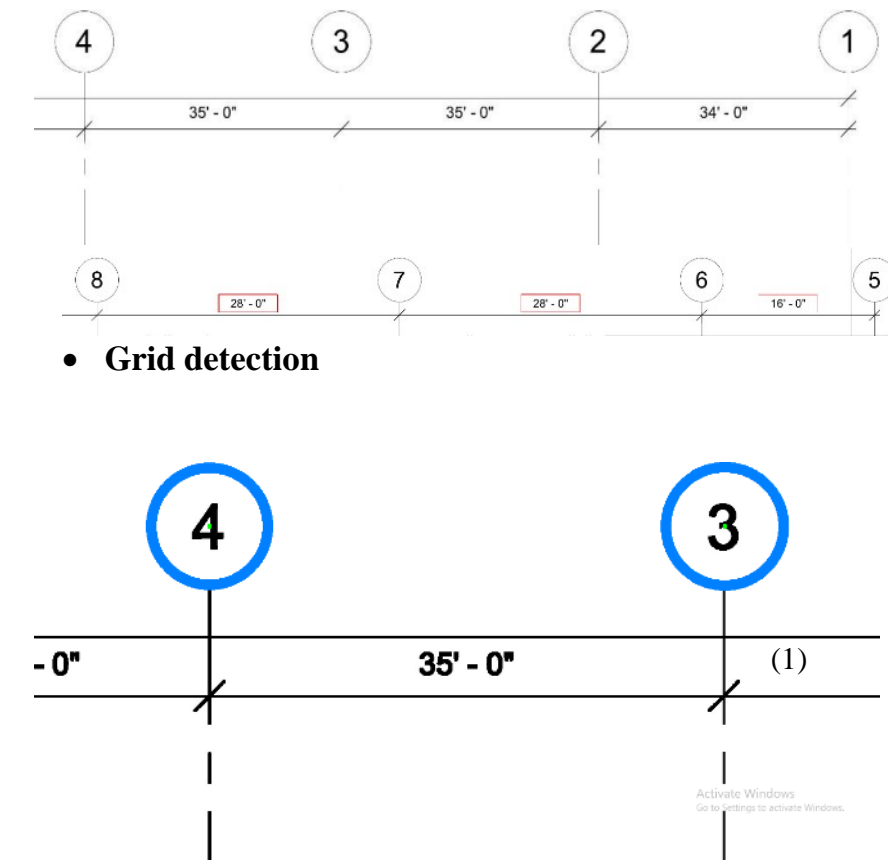

- Detect dimensions

\section{- Cropping}

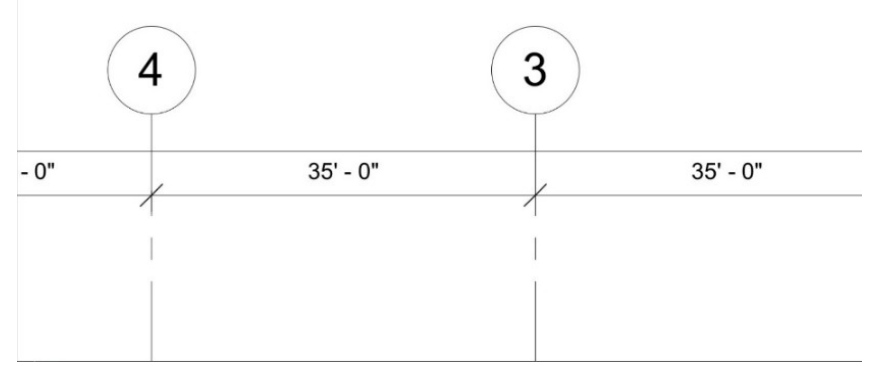

The second segment of this project is Structural Assembly Drawing Verification using CNN. The classifier is deployed on to the web portal that provides a features to upload new

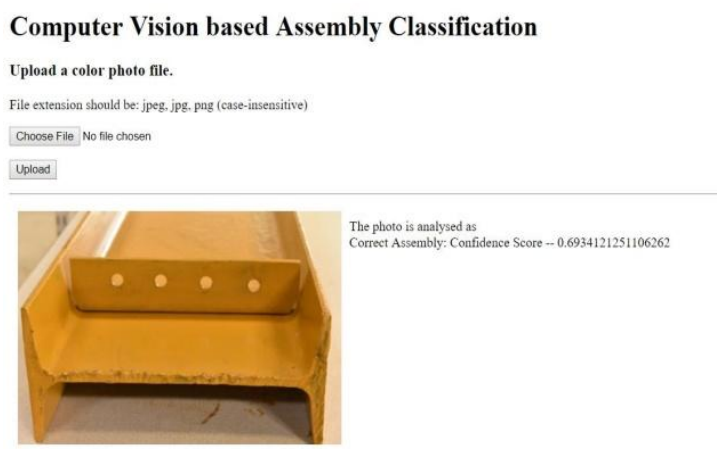

Fig 4.2(a): Correct assembly

assembly images for testing. Upon the classification being completed the web portal states whether the assembly is correct or there is error in assembly. Fig 4.2(a) shows the correct assembly. Fig 4.2(b) shows error in assembly.
- Grid detection

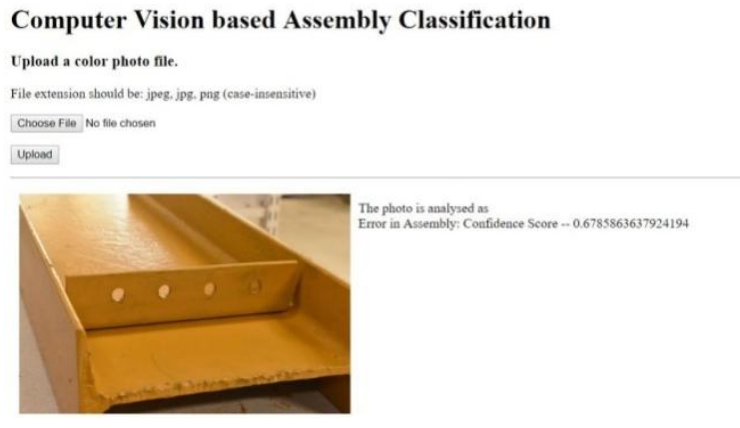

Fig 4.2 (b): Error in assembly

\section{CONCLUSION:}

CNNs model is found to be more effective with regard to efficiency and time over the other methods of classification.. This is because the high accuracy achieved by CNN in image classification and ability to handle images with multiple layers. In this paper inception v3 and transfer learning approach is used to classify the assembly images. Web portal upon classification of images provides a confidence score. Cropping Confidence score is with respect to the accuracy of the uploaded assembly images with the trained data set. Table 1 depicts the confidence score with respect to Fig 4.2 (a) and (b).

Table I: Confidence Score of assembly images

\begin{tabular}{|c|c|c|}
\hline & $\begin{array}{c}\text { Confidence Score for } \\
\text { Correct Assembly }\end{array}$ & $\begin{array}{c}\text { Confidence score } \\
\text { foe Error in } \\
\text { Assembly }\end{array}$ \\
\hline $\begin{array}{l}\text { Fig } \\
4.2 \text { (a) }\end{array}$ & 0.6934 & - \\
\hline Fig \\
4.2(b)
\end{tabular}

\section{REFERENCES}

1. Tianmei Guo, Jiwen Dong ,Henjian Li'Yunxing Gao "Simple Convolutional Neural Network on Image Classification" World Academy of Science, Engineering and Technology 2017.

2. Belen Ferrer, Juan C. Pomares, Ramon Irles, Julian Espinosa, and David Mas "Image processing for safety assessment in civil engineering" International Conference on Computational Intelligence and Networks 2018.

3. Pavol Bezak "Building Recognition System Based on Deep Learning" 2016

4. Arthur Shi ,Xiao-Hua Yu" Structural Damage Assessment Using Artificial Immune Systems and Wavelet Decomposition" 2018

5. Annegreet van Opbroek, M. ArfanIkram, MeikeW. Vernooij, Marleen de Bruijne," Transfer Learning Improves Supervised Image Segmentation Across Imaging Protocols"IEEE Transactions on Medical Imaging (Volume: 34, Issue: 5 , May 2015)

6. Pooja Sharma,Shanu Sharma," Image Processing based Degraded Camera Captured Document Enhancement for Improved OCR Accuracy" 6th International Conference - Cloud System and Big Data Engineering (Confluence)2016

7. RohitSaluja ;DevarajAdiga ; ParagChaudhuri ; Ganesh Ramakrishnan ; Mark Carman."Error Detection and Corrections in Indic OCR Using LSTMs" 14th IAPR International Conference on Document Analysis and Recognition (ICDAR)2017

8. HanshengLei, MandeShen," Improving OCR Performance with Background Image Elimination" 12th International Conference on Fuzzy Systems and Knowledge Discovery (FSKD)2015.

Published By:

Blue Eyes Intelligence Engineering

\& Sciences Publication

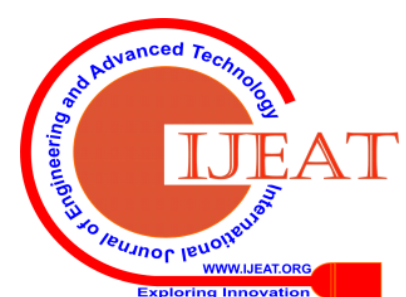


9. Weiheng ZHU1, Yuanfeng LIU2, Liang HAO1," A novel OCR approach based on document layout analysis and text block classification" 12th International Conference on Computational Intelligence and Security2016.

10. M.Savastano, L. Lecce, F.Marulo,C.Sansone," Use of Artificial Neural Networks for Optimal Sensing in Complex Structures Analysis" Conference Proceedings. 10th Anniversary. /94. Advanced Technologies in I \& M. 1994 IEEE Instrumentation andMeasurement Technolgy Conference (Cat. No.94CH3424-9)

11. J. Blasco, S. Cubero, J. Gomez-Sanchis, P. Mira, E. Molto, "Development of a machine for the automatic sorting of pomegranate (Punicagranatum) arils based on computer vision", J. Food Eng., vol. 90, no. 1, pp. 27-34,2009.

12. R. Kozma and M. Kitamura, A. Malinowski and J.M. Zurada"On Performance Measures of Artificial Neural Networks Trained by Structural Learning Algorithms" Proceedings 2015Second New Zealand International Two-Stream Conference on Artificial Neural Networks and Expert Systems.

13. Xiao-Hua Yu, Arthur Shi," Structural Damage Assessment Using ArtificialImmune Systems and Wavelet Decomposition" International Joint Conference on Neural Networks (IJCNN) 2017

\section{AUTHORS PROFILE}

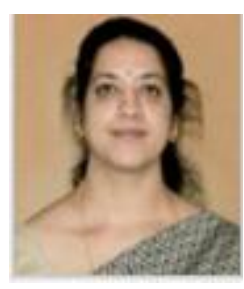

Dr. Padmashree $\mathbf{S}$ is working as Professor, Department of ECE, GSSS Institute of Engineering and Technology for women, Mysuru. She is a senior ieee member. She has got 28 years of teaching experience.She has obtained her Bachelor's degree in Electronics and communication in the year 1993 and has secured first rank in Mtech Bio Medical instrumentation. She has obtained her $\mathrm{PhD}$ degree in Image Processing and has published around 20 papers in national and international journals. She has presented 60 papers in national and international conferences. She has given lectures and technical talks in the area of Image processing , machine learning and computer networks. Her area of interests include Medical Image Processing, Computational Intelligence, Machine learning, Deep learning.

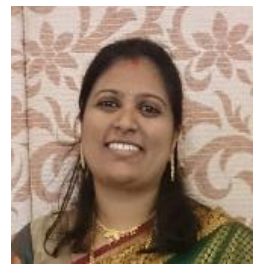

Sushma SJ is working as Associate Professor, Department of ECE, GSSS Institute of Engineering and Technology for women, Mysuru. She is a senior ieee member. She has got 20 years of teaching experience. She has obtained Bachelor of Engineering from Manglore University in the year 2001. In 2007 she obtained Master of Technology from Visveswaraya Technological University, Belagavi. Currently pursuing Ph.D. a Visveswaraya Technological University, Belagavi, India. She also possess diploma in psychological counsellor and is a active She has published around 18 papers in national and international journals and presented 72 papers national and international conferences. She has given lectures and technical talks in the area of machine learning and computer networks. Her area of interests include Medical Image Processing, Computational Intelligence, Machine learning, Deep learning and Computer Networks.

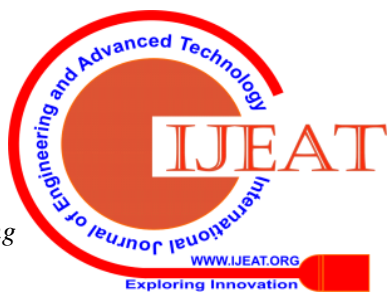

